\title{
ELECTROENCEPHALOGRAPHIC INDICATORS IN DEFAULT MODE, HABITUATION, AND VISUAL-MOTOR ASSOCIATION IN FEMALE COLLEGE STUDENTS
}

\author{
Héctor Brust-Carmona ${ }^{1 *}$, Yessica Martínez-Serrato ${ }^{1}$, Ana Sánchez-Quezada ${ }^{2}$, \\ Blanca Flores-Avalos ${ }^{2}$, Javier Alducin-Castillo ${ }^{3}$ ANd OsCar Yáñez-SuareZ ${ }^{4}$ \\ ${ }^{1}$ Department of Neurosciences, EEG Laboratory, Research Division, Instituto Nacional de Rehabilitación (INR LGII); \\ ${ }^{2}$ Neurophysiology Department, Electrodiagnosis Service, Instituto Nacional de Rehabilitación (INR LGII); \\ ${ }^{3}$ Department of Electronics, Instrumentation Section, Universidad Autónoma Metropolitana, Azcapotzalco; \\ ${ }^{4}$ Department of Neuroimaging, Universidad Autónoma Metropolitana, Iztapalapa. Mexico City, Mexico
}

\begin{abstract}
Background: Oscillations, action, and postsynaptic potentials in glial-neuronal ensembles integrate the spectral power (SP) of electroencephalographic (EEG). Our objective is to propose SP indicators of healthy brains (control groups) based on the default mode and their modifications by habituation and visual-motor association (VM-asso), to support diagnostic and rehabilitation processes. However, important differences seem to exist between men and women. Therefore, we aim to analyze EEG characteristics in a female sample. Subjects and Methods: In the current study, EEG was recorded at rest with closed eyes before and during repeated photostimulations (RPh) and before (pre) and during association of RPh with switch pressing (VM-asso) in 70 female adults. EEG was analyzed using UAM/INR software, which removes artifacts, identifies corresponding signals, selects 20 samples (2-s) from each condition, applies Welch's periodogram to calculate and average the absolute power (AP; AAPs) of $z, \theta, \alpha$, and $\beta$, before and during learning, and emits data to a spreadsheet. Differences in each condition were evaluated using non-parametric tests. Results: The AAPs were different; in habituation, $z$ and $\theta$ increased significantly in fronto-frontal (FF) and frontocentral and decreased in the other leads. During association, they increased in FF and decreased in the other three regions. $\alpha$ AAP decreased in all leads except in FF during habituation, and the decrement was lower in the association. $\beta$ AAP increased in all leads during both learning conditions. Conclusion: The SP and the topographic distribution are descriptive parameters of habituation and VM-asso; we propose them as EEG indicators.
\end{abstract}

Key words: Narrowband electroencephalographic in habituation and association. Electroencephalographic descriptive parameters. Habituation alpha inhibition. Visual-motor association. Beta increases. Evoked/event-related oscillation.

Corresponding author:

*Héctor Brust-Carmona

Laboratorio EEG, Dirección de Investigación

Instituto Nacional de Rehabilitación

Piñones, 29

Col. Rancho de los Colorines, Del. Tlalpan

C.P. 14386, Ciudad de México, México

Received for publication: 26-01-2017

E-mail: brusthector@gmail.com

Accepted for publication: 14-06-2017

doi: $10.24875 /$ RIC.17002309 


\section{INTRODUCTION}

Our main objective is to describe the electroencephalographic (EEG) spectral power (SP) parameters to identify and propose indicators (possible EEG biomarkers) in normal subjects of different ages and similar economic-cultural situations to use them to compare EEG parameters of the healthy brain or that disorganized by neurological diseases. To this end, we have been publishing the results of samples of both men and women ${ }^{1,2}$. However, there are publications that indicate important differences between women and men that could lead to misinterpretation in clinical diagnosis ${ }^{3,4}$. Beta frequency has been shown to be different in women than in men when responding to faces ${ }^{5}$. A recent study in a very large sample describes an increased frequency and higher absolute power (AP) of alpha range in women ${ }^{6}$. Therefore, the aim of the current work is to describe EEG characteristics in a sample of adult women.

The graphic representation of the AP of diverse frequency bands of the SP density results in topographical profiles. A set of profiles is proposed as a basis to regulate the mechanisms of behavior ${ }^{7-9}$.

The cognitive processes that result from the activity of multiple glial-neuronal ensembles constituted in networks are tuned by the electrotonic diffusion of the field potentials, and in turn, synchronize neuronal excitability. Varela et al. ${ }^{10}$ proposed that synchronized, rhythmic changes in excitability are relevant for sustaining long-range neuronal communication, complementing with neuronal coding of short and long connections. Furthermore, the neighboring neurons are also tuned by the electrotonic diffusion of membrane oscillations with the possible participation of astrocytes; both processes constitute a communications system among circuits and an integrating process that regulates the balance between excitatory and inhibitory functions of neuronal-glial ensembles ${ }^{11,12}$.

In this way, multiple glial-neuronal ensembles become specialized as cortical columns and layers in subcortical nuclei with a given topographical organization ${ }^{13}$. Further, these organizational networks can be related to specific behaviors such as hand movements ${ }^{14}$. Through this process, a greater number of attuned neurons results in a field potential with greater intensity ${ }^{15,16}$.
Repetition of these processes generates oscillations at a specific frequency and power that will dynamically integrate the glial-neuronal network in multiple brain areas. We propose that normal function occurs within a given range of AP of a glial-neuronal circuit. Thus, the behavior will be normal within a specified range of AP and tuned to one or several possible frequencies ${ }^{17}$. In the past scenarios, the subject evoked oscillations, bursts of action, and synaptic potentials in multiple glial-neuronal ensembles, which are within the networks that are integrated in a SP, generating the "basal" EEG condition, also described by functional magnetic resonance imaging analysis ${ }^{18}$.

We can infer the morphofunctional integrity of neurons and their connections by analyzing the EEG's AP and the attuned profiles of those frequencies while the subject is at rest with closed eyes, as proposed by many authors, including Catani and Ffytche ${ }^{19}$.

The profiles can be obtained by quantifying the spectral power density of the EEG in four frequency bands $(\gamma, \theta, \alpha$, and $\beta$ ) which integrate the basal, i.e., default condition, as well as when these bands are modified by photostimulation alone or by its association with a specific hand movement.

We present the characteristics of the EEG's SP with its topographic distribution in the default condition, as well as during habituation and visual-motor association (VM-asso), in a sample of 70 female college students.

\section{SUBJECTS AND METHODS}

Different groups of students were briefed on the study goals, procedures, and risks. Those interested in participating signed an informed consent letter issued by the National Institute of Rehabilitation of Mexico. The Institutional Research and Ethics Committee, following the guidelines of the Declaration of Helsinki, approved the research with protocol number 10/13.

A total of 70 healthy female college students (average age $21.5 \pm 2.5$ years) with similar economic and social status participated in the study. All recordings took place in a Faraday chamber that was dimly illuminated and sound attenuated. Each participant was placed in dorsal decubitus resting position and asked to remain 
as motionless as possible without falling asleep (we continuously observed both behavior and recordings to verify the "awake" condition). Recordings were made with an EEG (Nicolet one) with the electrodes placed at the $10 / 20$ distribution, and special attention was paid to the interelectrode distance. The impedance was maintained between 5 and $10 \mathrm{k} \Omega$ for the entire study. Recording electrodes were placed on the outer side of the eyes to register the eye and eyelid movements. For analysis, bipolar leads were used in the parasagittal left (Fp1F3, F3C3, C3P3, and P3O1) and right (Fp2F4, F4C4, C4P4, and P4O2) hemispheres. The EEG recording paradigm was as follows: A no-task and eyes-closed condition followed by a 20-times repeated photostimulation ( $\mathrm{RPh}, \mathrm{Habi}$ ), each at $5 \mathrm{~Hz}$, during $2 \mathrm{~s}$ at variable intervals of 20-25 s, applied with a Nicolet lamp placed at $70 \mathrm{~cm}$ from the subject's face (the subjects were unaware of the application of photostimulations). Then, second eyes closed condition was recorded followed by a similar photostimulation procedure but with the addition of an indication to press a switch (normally open) when perceiving the light and to hold the switch until the end of the series of flashes (VM-asso).

\section{Statistical analysis}

A computer program (UAM/INR) was used to remove eyelids, ocular, and electromyography (EMG) artifacts using the blind separation sources and the algorithm FastICA ${ }^{20}$. It eliminated electromorphograms with intensities higher than two standard deviations, by applying Shapiro-Wilk tests with windows of $1 \mathrm{~s}$ for each independent component ${ }^{21}$. Then, the program identified the flashes' signal and took 20 pre-stimulation samples (pre-Habi) of 2-s duration and samples from each series of 20-RPh. The software identifies the flashing signal, takes 20 pre-stimulation samples (pre-VM-asso), as well as the signal of voluntary motor response, and takes 20 samples of $\mathrm{VM}$-asso and measures their response latency.

By estimating the power spectral density using Welch's method (with $256 \mathrm{~Hz}$ sample rate), the program integrates the AP data per frequency $(\delta[1.6-4 \mathrm{~Hz}], \theta$ [4.5-8 Hz], $\alpha[8.5-13 \mathrm{~Hz}]$, and $\beta[13.5-30 \mathrm{~Hz}])$, calculates the averages of the AP (AAP) for each of the samples per frequency and lead, and transfers the data into Excel $^{\circledast}$ spreadsheets. Statistically significant differences were calculated using ANOVA and KruskalWallis analysis corrected by Dunnett's test ( $p \leq 0.05$ ). The software used for these analyses was Prisma ${ }^{\circledR}$ (GraphPad Prism version 6.00 for Windows, GraphPad Software, La Jolla, CA, USA).

\section{RESULTS}

\section{Comparison of the AAP of the four frequencies in closed eye condition (default mode) to that obtained in the condition before RPh (habituation) and before VM-asso}

Fig. 1 shows the AAP of $z$ in parasagittal leads in the basal condition. Note, its significant decrement from fronto-frontal (FF) to fronto-central (FC) leads in the left hemisphere and from FF to centro-parietal (CP) in the right hemisphere (Fig. 1 - upper part), and the increase in parieto-occipital (PO) leads, in both hemispheres. Besides, a greater AAP was observed in the right FC and PO leads, indicating a significant interhemispheric asymmetry.

In the condition before RPh, we recorded lower AAP of $z$ as compared to those described for the basal condition. Differences among FF, FC, and CP were significant; the increase in $\mathrm{P} 3 \mathrm{O} 1$ was significant versus $\mathrm{C} 3 \mathrm{P} 3$, whereas the increase in $\mathrm{P} 4 \mathrm{O} 2$ was significant versus $\mathrm{F} 4 \mathrm{C} 4$ and $\mathrm{C} 4 \mathrm{P} 4$.

In the condition before the VM-asso, AAPs lower than in the basal condition were recorded, and the decrease in F3C3 and C3P3 was significantly different from that recorded in $\mathrm{F} 1 \mathrm{~F} 3$ and $\mathrm{P} 3 \mathrm{O} 1$; the decrease in $\mathrm{F} 4 \mathrm{C} 4$ was significant as compared to F2F4 and P4O2. The interhemispheric asymmetry was no longer recorded during both previous conditions (Fig. 1 - lower part).

Theta activity (AAP) in the basal condition is shown in Fig. 2. The AAP was similar in the first three leads, but it was higher in $\mathrm{P} 3 \mathrm{O} 1$, yielding significant differences with $\mathrm{F} 3 \mathrm{C} 3$ and $\mathrm{P} 3 \mathrm{O} 1$ and of $\mathrm{P} 4 \mathrm{O} 2$ with $\mathrm{Fp} 2 \mathrm{~F} 4$ and C4P4. Again, a larger AAP was recorded in C4P4 and $\mathrm{P} 4 \mathrm{O} 2$ as compared to the similar left leads, indicating significant interhemispheric asymmetry.

In theta activity before RPh, the AAP showed a discrete increase with respect to the basal activity and 
Figure 1. Average the absolute power (AAP) with standard deviations (SD) of $z$ in the basal condition (upper part), before Habi (middle part), and before visual-motor association (lower part). Note the AAP diminution, with an increase in differences among leads, which are significant for the two pre-conditions. On learning, no more interhemispheric asymmetry was recorded. Asterisks indicate significant differences ${ }^{*} \mathrm{p} \leq 0.05,{ }^{* *} 0.01,{ }^{* * *} 0.001,{ }^{* * * *} 0.0001$ (this applies to all similar figures).

DEC

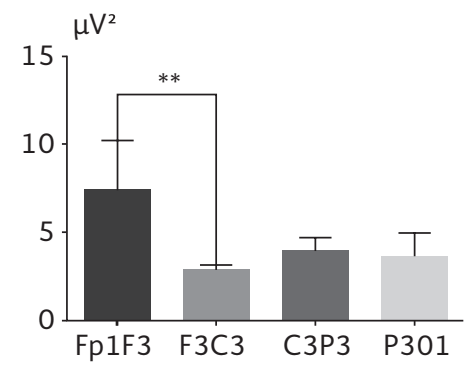

D pre habi

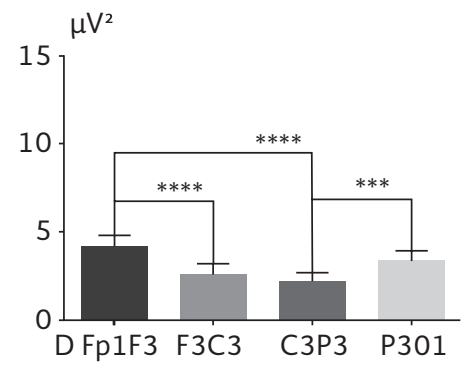

D pre vm

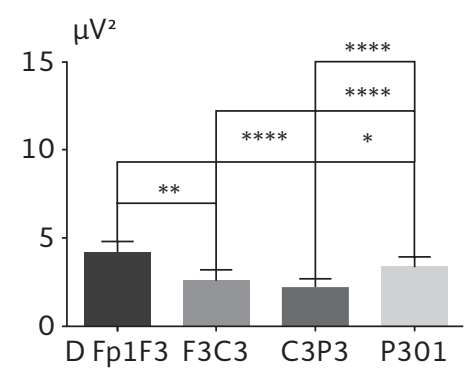

DEC

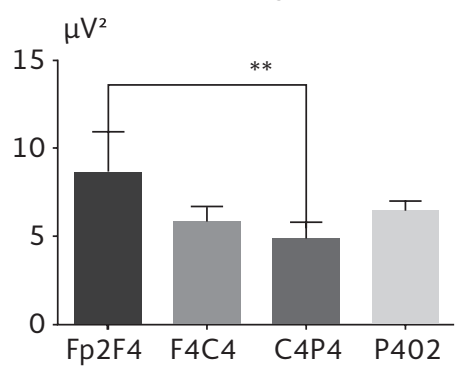

D pre habi

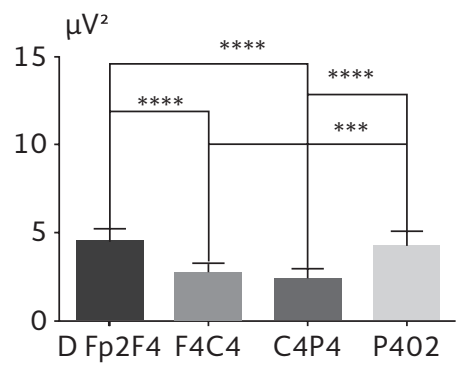

D pre vm

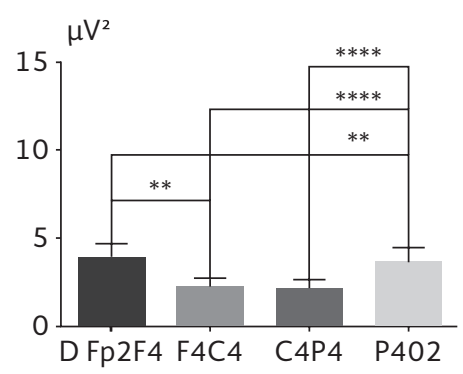

greater AAP in both $\mathrm{POs}$ so that it differed significantly from the other three leads (Fig. 2 - middle part).

In theta activity before VM-asso, discrete increases in the AAP of $\theta$ were recorded as compared to basal AAP, predominating in both POs, yielding a significant difference as shown in the lower part of Fig. 2. No asymmetry was recorded in the two conditions before Habi and VM-asso, as described for the basal condition.
Regarding alpha activity in the basal condition, AAP increased from frontal to occipital so that the difference was significant among FF, CP, and $P O$ in both hemispheres and between F4C4 and P4O2 (Fig. 3). In this frequency, in contrast to delta and theta, no interhemispheric asymmetry was recorded.

In alpha activity before RPh, the AAP increasing pattern from frontal to occipital was maintained, but in this condition, significant differences arose practically 
Figure 2. Average the absolute power (standard deviation) of $\theta$ in the basal condition (upper part), before habituation (pre-Habi; middle part), and before visual-motor associations (pre-VM-asso; lower part).
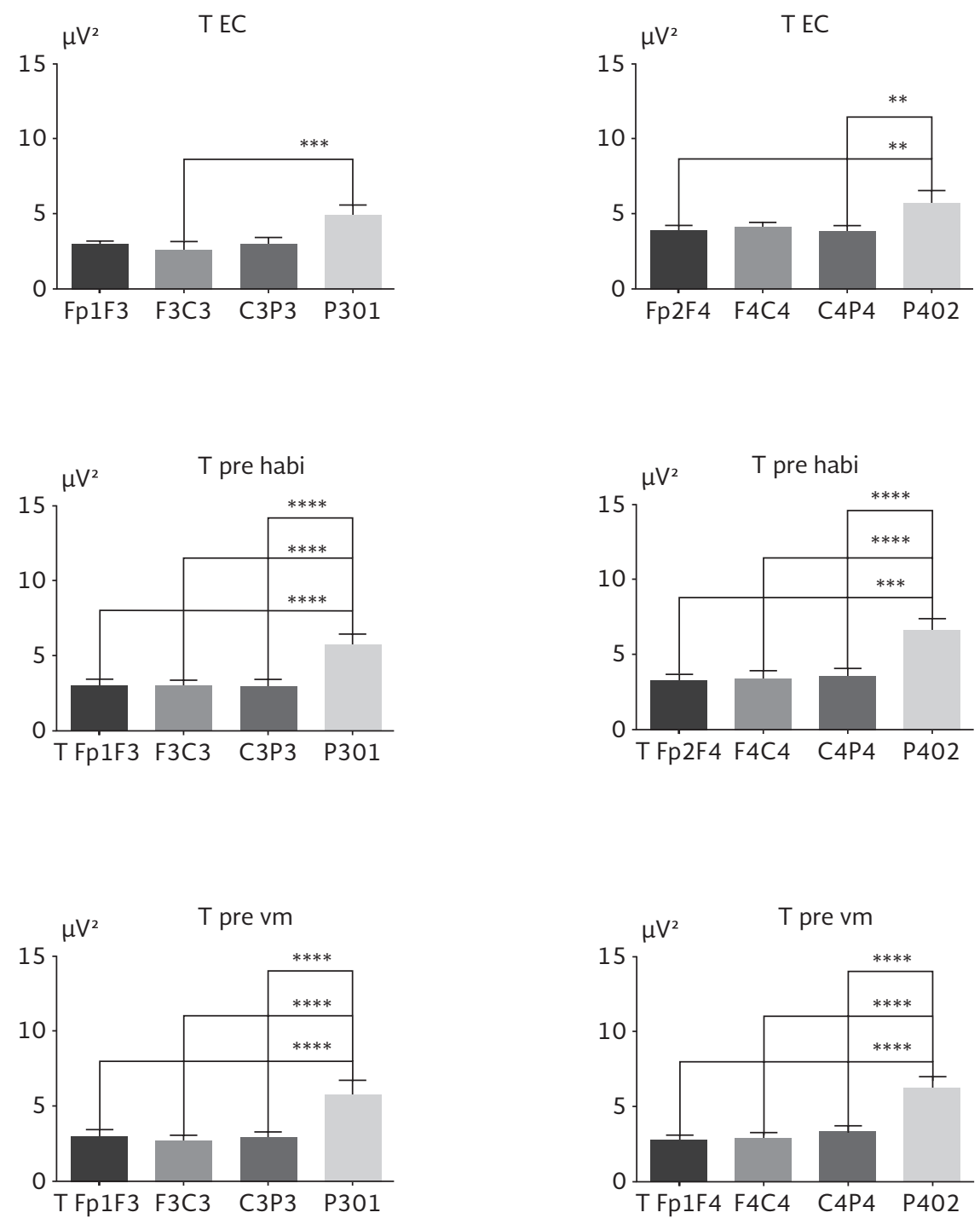

among all leads in both hemispheres, as shown in the middle part of Fig. 3 .

Alpha activity before VM-asso was very similar to that recorded in the pre-RPh condition (Fig. 3 - lower part).

Regarding beta activity in basal condition, the AAP diminished significantly in the left hemisphere from $\mathrm{Fp} 1 \mathrm{~F} 3$ to $\mathrm{F} 3 \mathrm{C} 3$ but increased in P3O1; thus, it was significantly different from $\mathrm{F} 3 \mathrm{C} 3$, whereas in the right hemisphere, $\beta$ decreased from Fp2F4 to $\mathrm{F} 4 \mathrm{C} 4$ and to $\mathrm{C} 4 \mathrm{P} 4$ but increased in $\mathrm{P} 4 \mathrm{O} 2$, giving a significant difference from F4C4 to C4P4. In this frequency, a higher voltage was recorded in $\mathrm{F} 4 \mathrm{C} 4$ than in $\mathrm{F} 3 \mathrm{C} 3$ and in $\mathrm{P} 4 \mathrm{O} 2$ than in $\mathrm{P} 3 \mathrm{O} 1$, yielding a significant asymmetry.

Beta activity in pre-RPh showed a similar pattern described for the basal condition but at least intensities, and the AAP difference becomes significant in the left hemisphere between $\mathrm{C} 3 \mathrm{P} 3$ and P3O1. In the right hemisphere, the AAP diminution in Fp2F4 eliminates the significant difference with respect to F4C4 recorded in the basal condition (Fig. 4 - middle part).

For beta activity in the pre-VM-asso condition, the recorded pattern followed the same characteristics 
Figure 3. Average the absolute power (standard deviation) of $\alpha$ in the basal condition (upper part), before habituation (pre-Habi; middle part), and before visual-motor associations (pre-VM-asso; lower part).
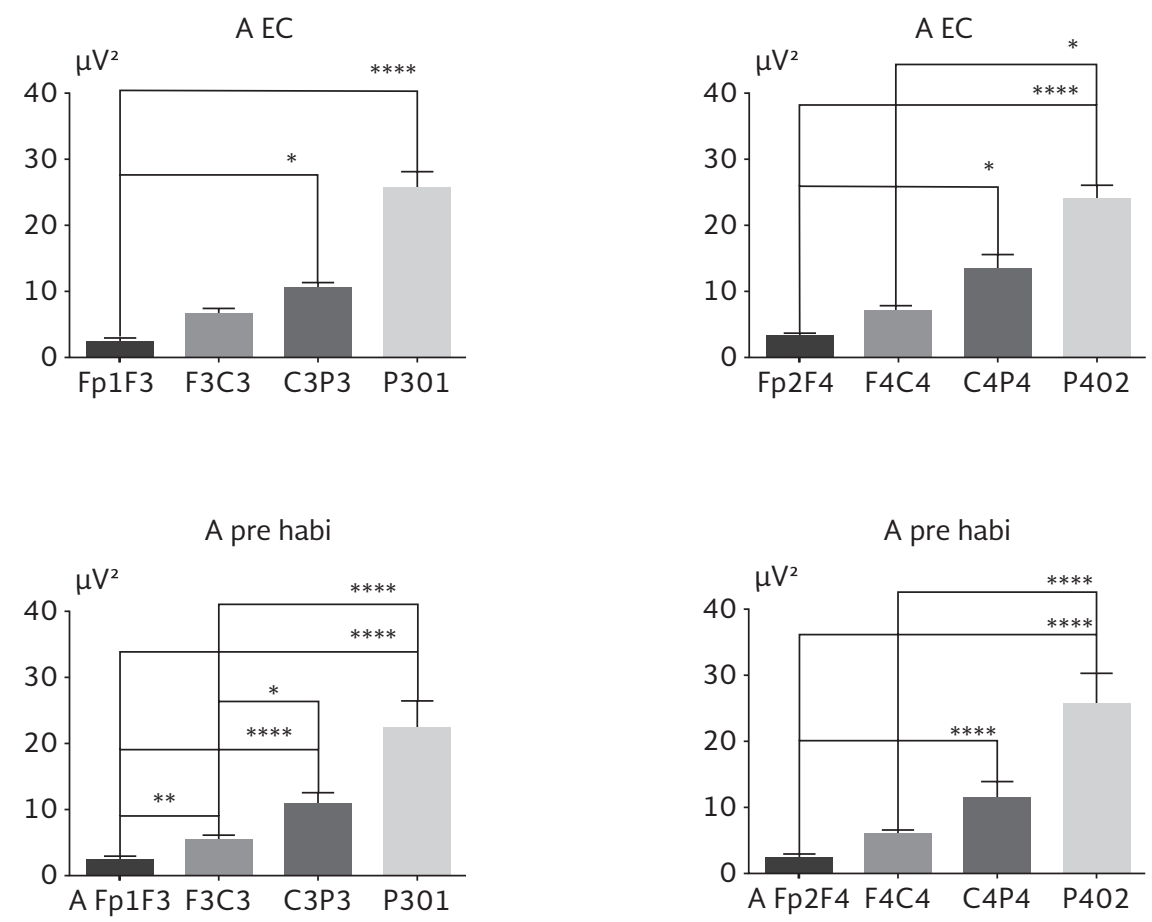

A pre vm

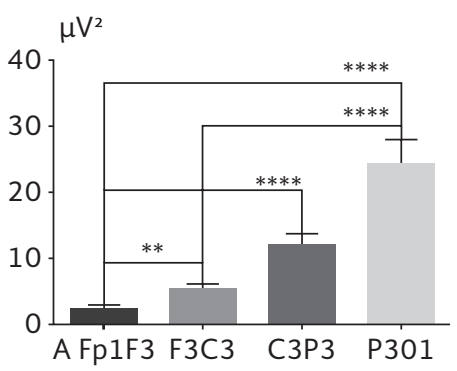

A pre vm

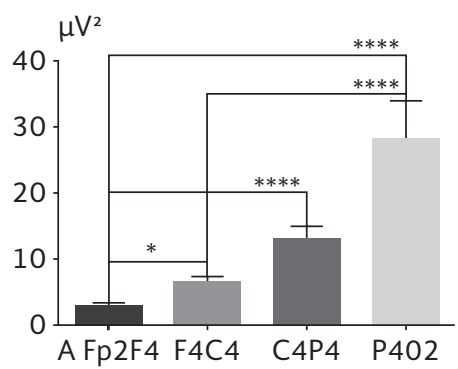

described for the basal condition except that the significant difference between leads FF and FC was maintained in both hemispheres (Fig. 4 - Lower part).

\section{Modification of the AAP of $\bar{\gamma}, \boldsymbol{\theta}, \alpha$, and $\beta$ during habituation and $\mathrm{VM}$-asso as compared to the before condition}

During habituation, the AAP of $z$ increased in FF and FC of both hemispheres and diminished in the other two leads. Similar changes occurred during VM-asso; however, the AAP's decrease was lower than that recorded during habituation (Fig. 5). The AAP of $\theta$ increased in FF of both hemispheres during both habituation and VM-asso; the change was higher during VM-asso. In all other leads, the $\theta$ AAP diminished during both learning conditions, with less decrement in associations, particularly in PO (Fig. 5).

During habituation, the AAP of $\alpha$ increased in FF of both hemispheres and diminished in all other leads. During associations, $\alpha$ diminished in all leads, as shown in Fig. 6. 
Figure 4. Average the absolute power (standard deviation) of $\beta$ in the basal condition (upper part), before habituation (pre-Habi; middle part), and before visual-motor associations (pre-VM-asso; lower part).
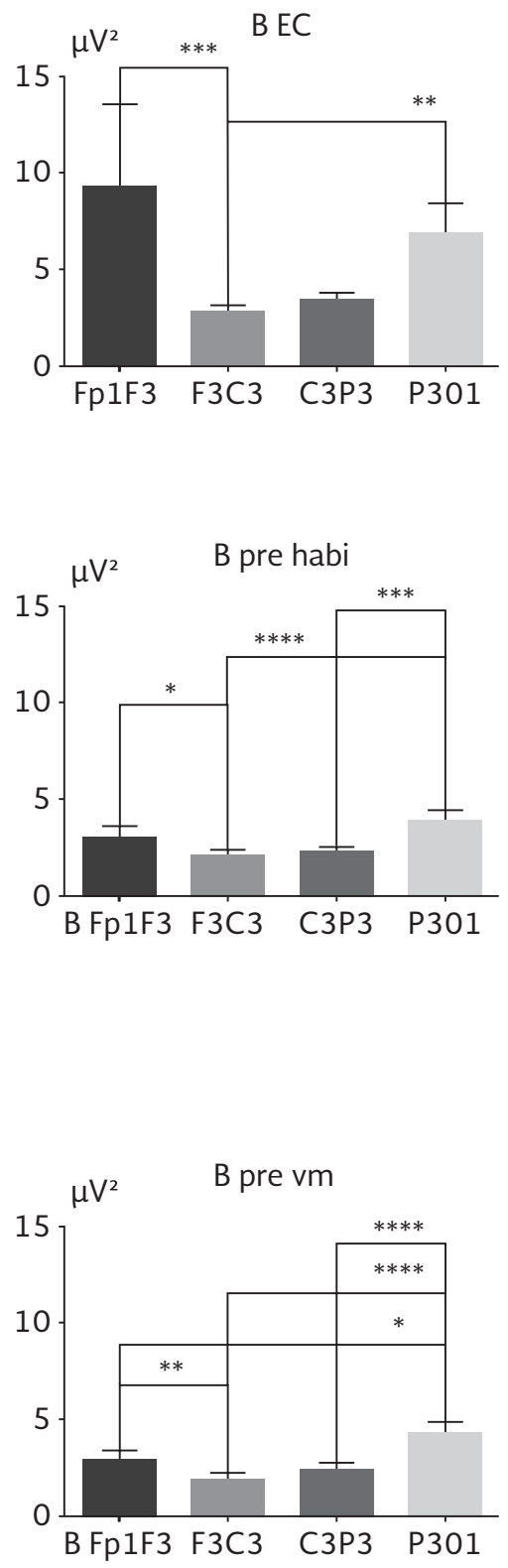
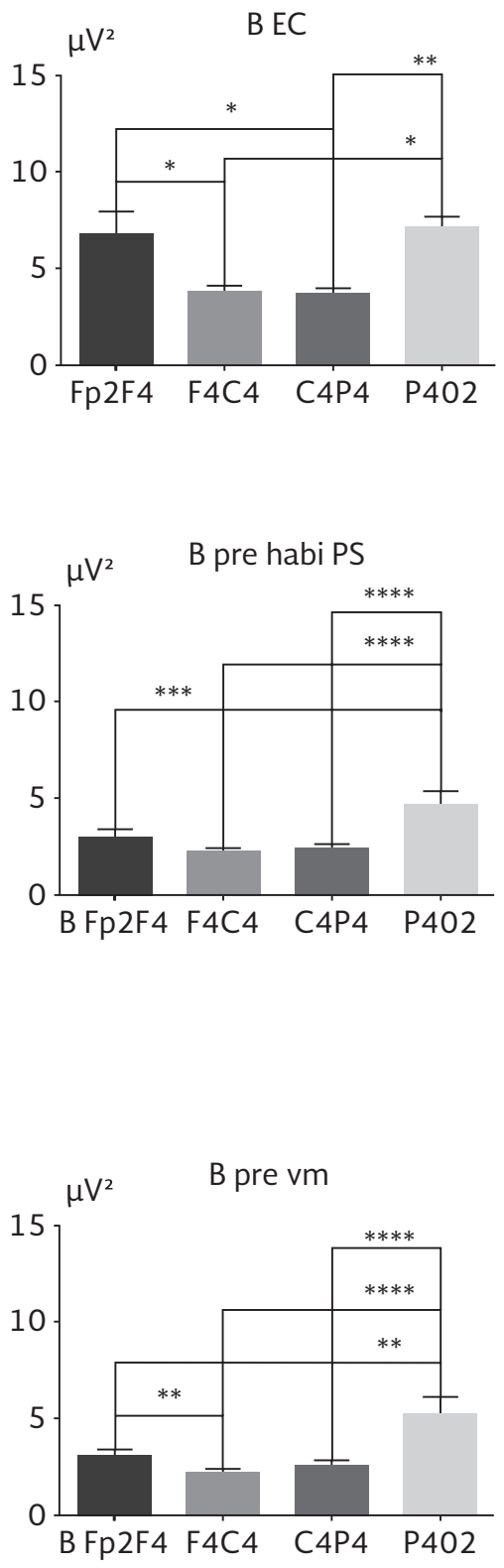

The AAP of $\beta$ increased significantly in all leads, except in $\mathrm{P} 4 \mathrm{O} 2$, during habituation; however, during associations, the increase was only significant in $\mathrm{CP}$ of both hemispheres (Fig. 6).

\section{DISCUSSION}

Cognitive neuroscience now leaves little or no doubt that cognitive processes require the transient integration of numerous, widely distributed, constantly interacting areas of the brain.

The most plausible mechanism for such a large-scale integration is the formation of dynamic links by oscillatory synchrony as well as bursts of action potentials generating multiple frequency bands in a specific range of AP in the brain network, which participate in sensation, perception, memory, as well as somatic and vegetative responses ${ }^{1,22-24}$. 
Figure 5. Modifications of the average the absolute power (AAP) of $z$ (upper part) and $\theta$ (lower part) in both preconditions and during habituation and visual-motor association (VM-asso) (A). Both learnings induce the AAP increase of both frequencies in fronto-frontal (FF) and fronto-central but reaching statistical significance only during VM-asso in FF; in all other leads, they diminished.
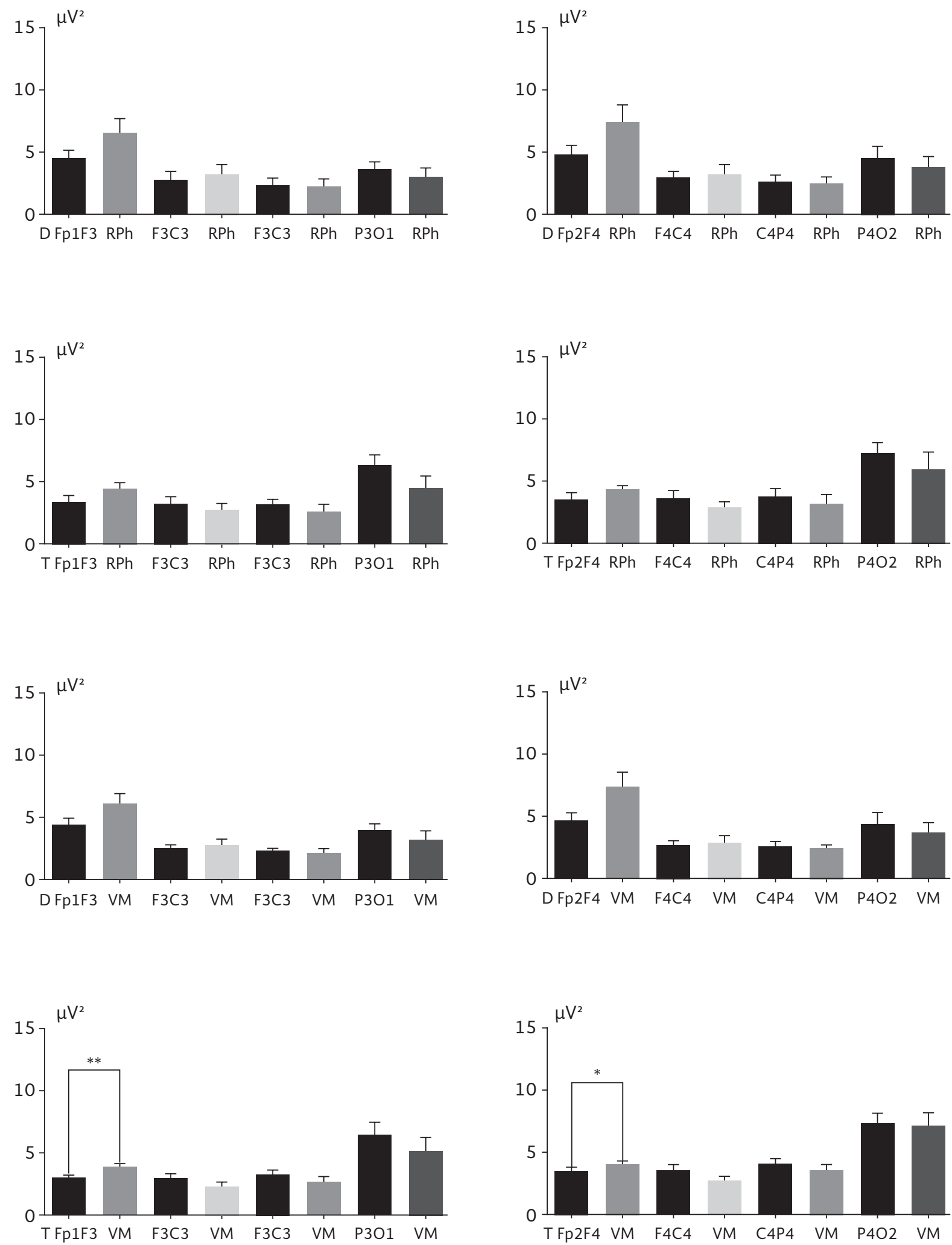
Figure 6. Modifications of the average the absolute power of $\alpha$ (upper part) and $\beta$ (lower part) in both preconditions and during habituation and visual-motor association. Note the increase in alpha in fronto-frontal, and the greater increase of beta during habituation.
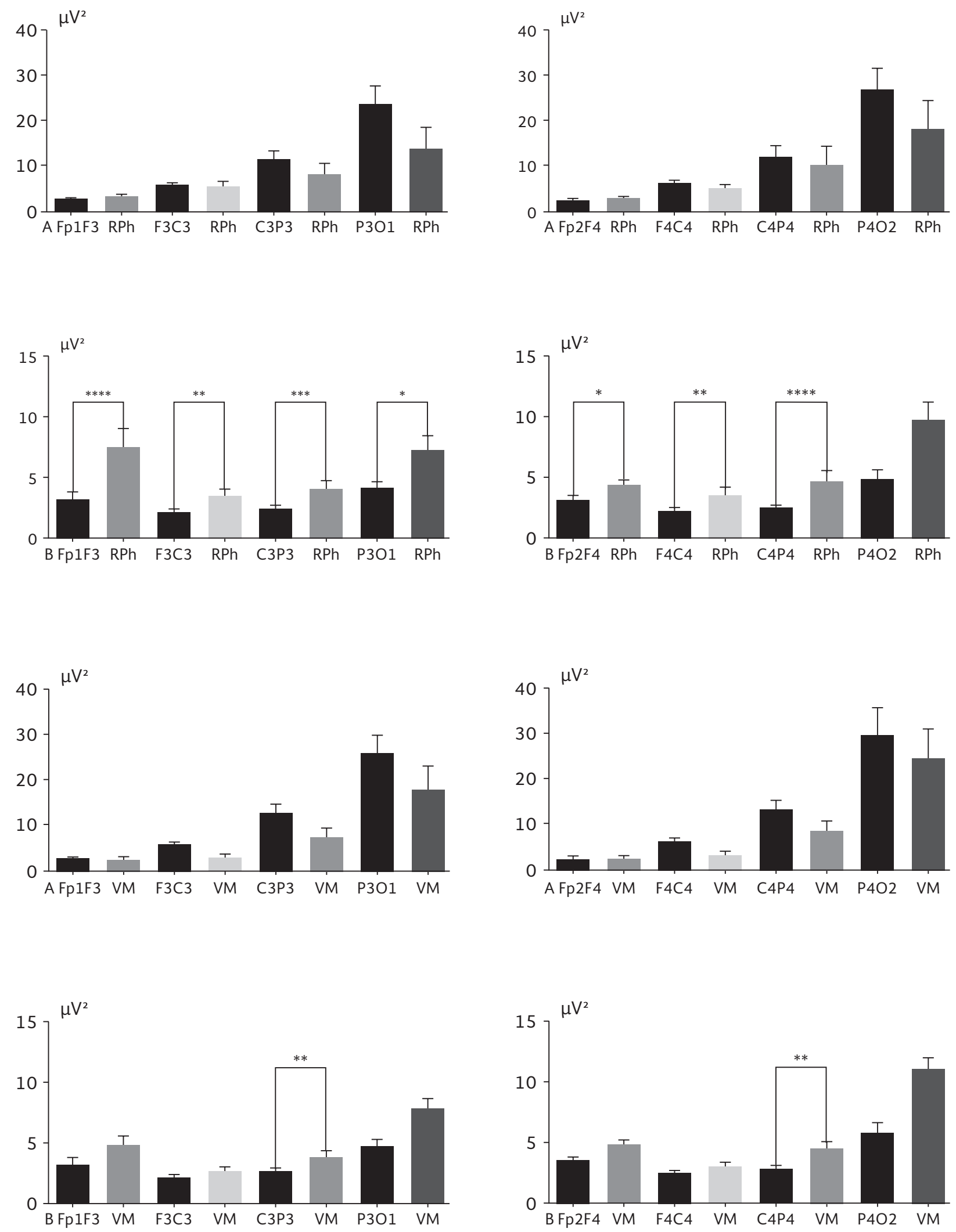
Oscillations in $\delta, \theta, \alpha$, and $\beta$ frequency bands are involved in a broad variety of cognitive processes ${ }^{25}$.

Thus, the average AP (AAP) of the four analyzed frequencies behaved differently in each cortical region before and during habituation (RPh) or before and during VM-asso.

During habituation, the AAP of $\delta$ increased (synchronization) in FC cortical regions and decreased (desynchronization) in the other two parasagittal cortical regions. In addition, during VM-asso, synchronization occurred in FF and FC regions of both hemispheres, and desynchronization was recorded in the other two regions. These spectral $\delta$ power modifications likely participate in controlling the neurovegetative activity that occurs as a part of the responses in both learning processes. The synchronization of $\delta$ could represent an inhibitory process, as proposed for $\alpha^{25}$, suggesting that the $\delta$ rhythm contributes to the modulation of those circuits intervening in vegetative responses ${ }^{26}$; however, the activity increment in the medial and posterior cortical region could be related to its participation in attention, sensory, and/or motor integration processes $^{27-29}$. Furthermore, other studies on human $\delta$ $(0.5-3.5 \mathrm{~Hz})$ have shown its involvement in perception, attention, learning, decision, and working memory ${ }^{30}$.

These interpretations pose a dilemma, as $\delta$ is the EEG activity described most frequently in the presence of cerebral lesions ${ }^{31}$. However, a different function could be an explanation in relation to the distribution of delta's power. For instance, a gradual decrease of $\delta$ occurs in healthy subjects as compared to subjects with mild cognitive impairment and Alzheimer's disease patients; furthermore, the decrease in the $\delta$ response is also significant in bipolar disorder and schizophrenia ${ }^{13}$.

Interestingly, during VM-asso, $\delta$ synchronization was recorded in frontal regions and desynchronization in $\mathrm{PO}$ regions with a trend toward significance. We were expecting larger changes induced by $\mathrm{VM}$-asso, and thus, we interpret that less inhibitory processes are necessary during VM-asso but higher in other cortical regions to support the attention processes.

The AAP of $\theta$ increased (synchronization, meaning inactivation of neuronal activity) during habituation as well as during VM-asso, and in FC regions with desynchronization in the $\mathrm{PO}$ regions (neuronal activation of $\theta$ frequency), this could be the way to become attuned to other glial-neuronal ensembles. Coupling of slowwave oscillations was found to occur over large distances, even between widely separated cortical areas, and to involve subcortical regions such as the thalamus or striatum ${ }^{32,33}$. Thus, we related the modification in theta activity to memory processes necessary in both types of learning.

The AAP of $\alpha$ showed a small increment in the FF region and diminished (desynchronization) in the other regions, during pre-Habi, and diminished in the four leads before VM-asso, compared to the basal condition. The $\alpha$ activation could be the neural process used to identify and analyze the meaning of the RPh and the expected scenario during association, resulting in feedback activation of the descending circuits, the reticular-thalamic-limbic-basal ganglia, as well as to facilitate motivation, vegetative, and motor networks responsible for the orientation reflex and sympathetic activation. All of these actions are manifestations of the descending regulatory function of $\alpha$ rhythm ${ }^{9,34,35}$.

In both types of simple learning, habituation, and VM-asso, the first stimulations produce $\alpha$ activation of different circuits of the visual pathway and the mesencephalic-reticular-thalamic-cortical system. This activation increases the state of vigilance, including the frontal cortical and limbic circuits of association that participate in the integration of the meaning of the sensory stimulation ${ }^{36-38}$. Similar desynchronization has been described as stimulus expectation ${ }^{39}$. We expected that, in the same scenario, the responses would decrease when repeating the stimulation without important changes in the organism's homeostasis, such as the orienting reflex. Thus, this information is rated as "non-significant," and the organism learns not to respond; this process was called "habituation" by Sokolov in $1963^{40}$.

Decreased $\alpha$-desynchronization (or $\alpha$-started synchronization) will occur during habituation, and during VM-asso, desynchronization will persist. However, the AAP was lower in both learning processes. Nevertheless, the average did not show the temporal evolution of the AP changes in each pre- and learning condition; hence, we applied linear regression analysis to establish the possible desynchronization/synchronization slope (activation/inactivation) during each pre- and learning process. In addition, the average of 
the other frequencies also masked the evolution of the AP in each pre-stimulation and during learning.

The AAP of $\beta$ increased in both habituation and VM-asso with a greater intensity during habituation; the latter could indicate either an increase in the attention filtering processes, attempting to concentrate the activity in specific networks to diminish the non-significant responses, or to retain the gating control of different circuits in the VM-asso. However, we were expecting higher $\beta$ modifications during VM-asso, but only a significant increment was observed in the right $\mathrm{CP}$ region, probably related to the motor response. The possible explanation of less change of beta activity is that, during the VM-asso, beta's gating activity has to be more localized to cortical regions. Furthermore, it is important to analyze the evolution of the AP in the 20 learning responses.

The descriptions presented herein provide support to the hypothesis that, when beta syntonizes in the different cortical regions, it coordinates the attention and sensory-perception processes and motor functions $^{32,38,41,42}$. Other authors have described this condition, as the manifestation of the up-down regulation of organisms ${ }^{43}$.

Tuning the different frequencies of the circuits involved in behavior could result from activation of thalamic nuclei and cortical-thalamic feedback circuits; this could also hold for the condition of suppressing non-specific, irrelevant responses. All of these changes are complemented with the "functional connection" to the pyramidal and extrapyramidal motor modules, which are concatenated in the regulatory processes of the still unknown reticular-thalamic-cortico-cortical-thalamic-basal nuclei; these are mechanisms that regulate the sensory-motor information in learning processes and are reflected in the modifications of the EEG power spectrum. In these circuits, the profile of the electrical activity could represent the characteristics of the different scenarios, similarly to what occurs during space representation ${ }^{44}$.

A limitation of the present study is that we did not identify the origin and sequence of the SP modifications. Therefore, we recognize the low capacity of the EEG to identify the source of electrical changes and the necessity to use other computer analyses, such as coherence. A major weakness is that EEG recordings represent changes in the local field potentials of thousands of neurons of diverse circuits, which may have different functions and times of participation that are inherent in the changes in the EEG rhythms. However, we insist that the quantitative EEG is more applicable than neuroimaging studies because it is safe, does not have medical contraindications, is easier to perform in different subjects (even children) and patient's conditions, and is less expensive.

For neurorehabilitation programs, recognizing the proposed markers will help to improve the diagnosis of acquired and chronic neurological diseases. For instance, Conway et al. $^{45}$ describe synchronization between motor cortex and spinal motoneuronal pool to maintain a motor task in man.

Brain oscillations in multiple frequency windows may be used as physiological markers for the recognition and progression of diseases or to improve rehabilitation effects, as shown in simple learning processes like habituation and association of a sensorial signal and a voluntary motor response. Spatial coherence in diseases may be helpful in analyzing integrative brain functions, and thus, are important for the future research.

\section{ACKNOWLEDGMENTS}

The authors would like to thank Ingrid Mascher for editorial assistance and Dr. Teodoro Flores for administrative support. This study was performed with support from CONACyT-Mexico, Salud-2011 No. 1161587.

\section{REFERENCES}

1. Brust-Carmona H, Valadez G, Flores-Ávalos B, et al. Potencia absoluta de oscilaciones corticales y su distribución topográfica en una muestra de adultos jóvenes en vigilia inactiva y en atención inespecífica. Rev Invest Clin. 2013;65:52-64.

2. Brust-Carmona H, Valadez G, Galicia M, et al. Desynchronization/ synchronization of lateral EEG rhythms during habituation to photostimulation in adults. Rev Invest Clin. 2013;65:436-44.

3. Aurlien $\mathrm{H}$, Gjerde IO, Aarseth $\mathrm{JH}$, et al. EEG background activity described by a large computerized database. Clin Neurophysiol. 2004;115:665-73.

4. Cantillo-Negrete J, Carino-Escobar RI, Carrillo-Mora P, et al. Gender differences in quantitative electroencephalogram during a simple hand movement task in young adults. Rev Invest Clin. 2016;68:245-55.

5. Hashemi A, Pino LJ, Moffat G, et al. Characterizing population EEG dynamics throughout adulthood. eNeuro. 2016;3. pii: ENEURO.0275-16.2016. eCollection 2016. 
6. Güntekin B, Basar E. Gender differences influence brain's beta oscillatory responses in recognition of facial expressions. Neurosci Lett. 2007;424:94-9.

7. Buzsáki G, editor. Coupling of systems by oscillations. Rhythms of the Brain. Oxford, UK: Oxford University Press; 2011. p. 334-56.

8. Singer W. Synchronization of cortical activity and its putative role in information processing and learning. Annu Rev Physiol. 1993;55:349-74

9. Basar E, Basar-Eroglu C, Karakas S, Schürmann M. Brain oscillations in perception and memory. Int J Psychophysiol. 2000;35:95-124.

10. Varela F, Lachaux JP, Rodriguez E, Martinerie J. The brainweb: Phase synchronization and large-scale integration. Nat Rev Neurosci. 2001;2:229-39.

11. Fries P. A mechanism for cognitive dynamics: Neuronal communication through neuronal coherence. Trends Cogn Sci. 2005;9:474-80.

12. Wyart V, Sergent C. The phase of ongoing EEG oscillations uncovers the fine temporal structure of conscious perception. J Neurosci. 2009;29:12839-41.

13. Basar E, Basar-Eroglu C, Güntekin B, Yener GG. Brain's alpha, beta, gamma, delta, and theta oscillations in neuropsychiatric diseases: Proposal for biomarker strategies. Suppl Clin Neurophysiol. 2013;62:19-54.

14. Grossberg S. How does the cerebral cortex work? Learning, attention, and grouping by the laminar circuits of visual cortex. Spat Vis. 1999;12:163-85

15. de Klerk CC, Johnson MH, Southgate V. An EEG study on the somatotopic organisation of sensorimotor cortex activation during action execution and observation in infancy. Dev Cogn Neurosci. 2015;15:1-10.

16. Pfurtscheller G. The cortical activation model (CAM). Prog Brain Res. 2006;159:19-27.

17. Michels L, Muthuraman M, Lüchinger R, et al. Developmental changes of functional and directed resting-state connectivities associated with neuronal oscillations in EEG. Neuroimage. 2013;81:231-42.

18. Wang $X \mathrm{~J}$. Neurophysiological and computational principles of cortical rhythms in cognition. Physiol Rev. 2010;90:1195-268.

19. Catani M, Ffytche DH. The rises and falls of disconnection syndromes. Brain. 2005;128:2224-39.

20. Hyvärinen A, Oja E. Independent component analysis: Algorithms and applications. Neural Netw. 2000;13:411-30

21. Shapiro SS, Wilk MB. An analysis of variance test for normality complete samples. Biometrika. 1965;52:591-611.

22. Lopes da Silva FH. Principles of neural coding from EEG signals. In: Quiroga Q, Panzeri S, editors. Principles of Neural Coding. Boca Raton, FL: CRC Press. Taylor \& Francis Group; 2013. p. 431-47.

23. Ben-Simon E, Podlipsky I, Arieli A, Zhdanov A, Hendler T. Never resting brain: Simultaneous representation of two alpha related processes in humans. PLoS One. 2008;3:e3984.

24. Kopell N, Kramer MA, Malerba P, Whittington MA. Are different rhythms good for different functions? Front Hum Neurosci. 2010;4:187.

25. Klimesch W, Sauseng P, Hanslmayr S. EEG alpha oscillations: The inhibition-timing hypothesis. Brain Res Rev. 2007;53:63-88.
26. Knyazev GG. EEG delta oscillations as a correlate of basic homeostatic and motivational processes. Neurosci Biobehav Rev. 2012;36:677-95

27. Harmony T, Fernández T, Silva J, et al. EEG delta activity: An indicator of attention to internal processing during performance of mental tasks. Int J Psychophysiol. 1996;24:161-71.

28. Bruns A, Eckhorn R. Task-related coupling from high-to low-frequency signals among visual cortical areas in human subdural recordings. Int J Psychophysiol. 2004;51:97-116.

29. Womelsdorf T, Fries P. Neuronal coherence during selective attentional processing and sensory-motor integration. J Physio Paris. 2006;100:182-93

30. Emek-Savas DD, Güntekin B, Yener GG, Basar E. Decrease of delta oscillatory responses is associated with increased age in healthy elderly. Int J Psychophysiol. 2016;103:103-9.

31. Thatcher RW, North DM, Curtin RT, et al. An EEG severity index of traumatic brain injury. J Neuropsychiatry Clin Neurosci. 2001;13:77-87.

32. Steriade M, Amzica F, Contreras D. Synchronization of fast (30-40 Hz) spontaneous cortical rhythms during brain activation. J Neurosci. 1996;16:392-417.

33. van Dijk H, Schoffelen JM, Oostenveld R, Jensen O. Prestimulus oscillatory activity in the alpha band predicts visual discrimination ability. J Neurosci. 2008;28:1816-23.

34. Engel AK, Fries $P$, Singer W. Dynamic predictions: Oscillations and synchrony in top-down processing. Nat Rev Neurosci. 2001;2:704-16.

35. Hummel F, Gerloff C. Larger interregional synchrony is associated with greater behavioral success in a complex sensory integration task in humans. Cereb Cortex. 2005;15:670-8.

36. Moruzzi G, Magoun HW. Brain stem reticular formation and activation of the EEG. Electroencephalogr Clin Neurophysiol. 1949;1:455-73.

37. Gvilia I. Underlying brain mechanisms that regulate sleep-wakefulness cycles. Int Rev Neurobiol. 2010;93:1-21.

38. Basar E, Güntekin B. A short review of alpha activity in cognitive processes and in cognitive impairment. Int J Psychophysiol. 2012;86:25-38.

39. Bastiaansen MC, Brunia CH. Anticipatory attention: An event-related desynchronization approach. Int J Psychophysiol. 2001;43:91-107.

40. Sokolov EN. Higher nervous functions; The orienting reflex. Annu Rev Physiol. 1963;25:545-80.

41. Wróbel A. Beta activity: A carrier for visual attention. Acta Neurobiol Exp (Wars). 2000;60:247-60.

42. Taylor AG, Goehler LE, Galper DI, Innes KE, Bourguignon C. Topdown and bottom-up mechanisms in mind-body medicine: Development of an integrative framework for psychophysiological research. Explore (NY). 2010;6:29-41.

43. Basar E, Düzgün A. The CLAIR model: Extension of Brodmann areas based on brain oscillations and connectivity. Int J Psychophysiol. 2016;103:185-98.

44. Grieves RM, Jeffery KJ. The representation of space in the brain. Behav Processes. 2017;135:113-31.

45. Conway B, Halliday D, Farmer S, et al. Synchronization between motor cortex and spinal motoneuronal pool during the performance of a maintained motor task in man. J Physiol. 1995; 489:917-24. 